Alison W. Loren, Perelman School of Medicine at the University of Pennsylvania, Philadelphia; Anthony J. Magdalinski, Private Practice, Sellersville, PA; Pamela B. Mangu, American Society of Clinical Oncology, Alexandria, VA; Lindsay Nohr Beck, LIVESTRONG Foundation's Fertile Hope Program, Austin, TX Kutluk Oktay, Innovation Institute for Fertility Preservation, New York Medical College, Rye and New York, NY; Lawrence Brennan, Oncology Hematology Care, Crestview Hills, KY; Ann H. Partridge, Dana-Farber Cancer Institute Boston, MA; Gwendolyn Quinn, Moffitt Cancer Center, Tampa, FL; and W. Hamish Wallace, Royal Hospital for Sick Children, Edinburgh, United Kingdom.

Published online ahead of print at www.jco.org on May 28, 2013.

A.L. and K.O. were Update Panel Co-Chairs.

Authors' disclosures of potential conflicts of interest and author contributions are found at the end of this article.

Copyright (C) 2013 American Society of Clinical Oncology. All rights reserved. No part of this document may be reproduced or transmitted in any form or by any means, electronic or mechanical, including photocopy, recording, or any information storage and retrieval system, without written permission by the American Society of Clinical Oncology

Corresponding author: Kutluk Oktay, $M D$, Institute for Fertility Preservation, New York Medical College, Rye, NY; email: koktay@fertilitypreservation.org.

For more information, contact American Society of Clinical Oncology, 2318 Mill Rd, Suite 800, Alexandria, VA 22314; e-mail: guidelines@asco.org.

C 2013 by American Society of Clinica Oncology

0732-183X/13/3119w-2500w/\$20.00

DOI: $10.1200 / J C O .2013 .49 .2678$

\title{
Fertility Preservation for Patients With Cancer: American Society of Clinical Oncology Clinical Practice Guideline Update
}

Alison W. Loren, Pamela B. Mangu, Lindsay Nohr Beck, Lawrence Brennan, Anthony J. Magdalinski, Ann H. Partridge, Gwendolyn Quinn, W. Hamish Wallace, and Kutluk Oktay

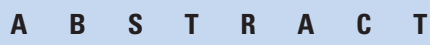

\section{Purpose}

To update guidance for health care providers about fertility preservation for adults and children with cancer.

\section{Methods}

A systematic review of the literature published from March 2006 through January 2013 was completed using MEDLINE and the Cochrane Collaboration Library. An Update Panel reviewed the evidence and updated the recommendation language.

\section{Results}

There were 222 new publications that met inclusion criteria. A majority were observational studies, cohort studies, and case series or reports, with few randomized clinical trials. After review of the new evidence, the Update Panel concluded that no major, substantive revisions to the 2006 American Society of Clinical Oncology recommendations were warranted, but clarifications were added.

\section{Recommendations}

As part of education and informed consent before cancer therapy, health care providers (including medical oncologists, radiation oncologists, gynecologic oncologists, urologists, hematologists, pediatric oncologists, and surgeons) should address the possibility of infertility with patients treated during their reproductive years (or with parents or guardians of children) and be prepared to discuss fertility preservation options and/or to refer all potential patients to appropriate reproductive specialists. Although patients may be focused initially on their cancer diagnosis, the Update Panel encourages providers to advise patients regarding potential threats to fertility as early as possible in the treatment process so as to allow for the widest array of options for fertility preservation. The discussion should be documented. Sperm and embryo cryopreservation as well as oocyte cryopreservation are considered standard practice and are widely available. Other fertility preservation methods should be considered investigational and should be performed by providers with the necessary expertise.

\section{J Clin Oncol 31:2500-2510. (C) 2013 by American Society of Clinical Oncology}

\section{INTRODUCTION}

In 2006, the American Society of Clinical Oncology (ASCO) published a clinical practice guideline on fertility preservation for adults and children with cancer. ${ }^{1}$ ASCO guidelines are updated periodically by a subset of the original Expert Panel. In October 2012, the Update Panel reviewed the results of a systematic review of the new literature and determined that although the recommendations remained the same (with the exception of adding oocyte cryopreservation as a standard practice, whereas in the previous guideline, it was still considered experimental), some information and tables needed to be updated. In terms of who is responsible for discussing fertility preservation, the original language used by ASCO has been revised: The word "oncologist" was replaced with "health care provider" to include medical oncologists, radiation oncologists, gynecologic oncologists, urologists, hematologists, pediatric oncologists, and surgeons, as well as nurses, social workers, psychologists, and other nonphysician providers.

\section{GUIDELINE QUESTIONS}

This clinical practice guideline addresses four overarching clinical questions: (1) Are patients with cancer interested in interventions to preserve fertility? 


\section{THE BOTTOM LINE}

\section{Fertility Preservation for Patients With Cancer: American Society of Clinical Oncology Clinical Practice Guideline Update}

\section{Intervention}

- Discuss the risk of infertility and fertility preservation options with patients with cancer anticipating treatment

\section{Target Audience}

- Medical oncologists, radiation oncologists, gynecologic oncologists, urologists, hematologists, pediatric oncologists, and surgeons, as well as nurses, social workers, psychologists, and other nonphysician providers

\section{Key Recommendations}

- Discuss fertility preservation with all patients of reproductive age (and with parents or guardians of children and adolescents) if infertility is a potential risk of therapy

- Refer patients who express an interest in fertility preservation (and patients who are ambivalent) to reproductive specialists

- Address fertility preservation as early as possible, before treatment starts

- Document fertility preservation discussions in the medical record

- Answer basic questions about whether fertility preservation may have an impact on successful cancer treatment

- Refer patients to psychosocial providers if they experience distress about potential infertility

- Encourage patients to participate in registries and clinical studies

\section{Adult Males}

- Present sperm cryopreservation (sperm banking) as the only established fertility preservation method

- Do not recommend hormonal therapy in men; it is not successful in preserving fertility

- Inform patients that other methods (eg, testicular tissue cryopreservation, which does not require sexual maturity, for the purpose of future reimplantation or grafting of human testicular tissue) are experimental

- Advise men of a potentially higher risk of genetic damage in sperm collected after initiation of chemotherapy

\section{Adult Females}

- Present both embryo and oocyte cryopreservation as established fertility preservation methods

- Discuss the option of ovarian transposition (oophoropexy) when pelvic radiation therapy is performed as cancer treatment

- Inform patients of conservative gynecologic surgery and radiation therapy options

- Inform patients that there is insufficient evidence regarding the effectiveness of ovarian suppression (gonadotropin-releasing hormone analogs) as a fertility preservation method, and these agents should not be relied on to preserve fertility

- Inform patients that other methods (eg, ovarian tissue cryopreservation, which does not require sexual maturity, for the purpose of future transplantation) are still experimental

\section{Children}

- Use established methods of fertility preservation (semen cryopreservation and oocyte cyropreservation) for postpubertal minor children, with patient assent, if appropriate, and parent or guardian consent

- Present information on additional methods that are available for children but are still investigational

- Refer for experimental protocols when available

\section{Methods}

- A comprehensive systematic review of the literature was conducted, and an Update Panel was convened to review the evidence and guideline recommendations

\section{Additional Information}

Data Supplements (including evidence tables) and clinical tools and resources can be found at http://www.asco.org/guidelines/fertility. 
(2) What is the quality of evidence supporting current and forthcoming options for preservation of fertility in males? (3) What is the quality of evidence supporting current and forthcoming options for preservation of fertility in females? (4) What is the role of the oncologist in advising patients about fertility preservation options? Special considerations addressing the fertility needs of children with cancer are also addressed.

\section{RECOMMENDATIONS, CLINICAL TOOLS, AND RESOURCES}

Table 1 provides the updated guideline recommendations. Clinical tools and resources, including links to related articles published in Journal of Oncology Practice and key Web sites, and Data Supplements are available at http://www.asco.org/guidelines/fertility, and a patient guide is available at http://www.cancer.net.

\section{METHODS}

The Update Panel included academic and community practitioners, in the fields of adult and pediatric oncology, obstetrics-gynecology, reproductive endocrinology and infertility, health services research, and psychosocial oncology, as well as a patient advocate (Appendix Table Al, online only). The Update Panel completed a review and analysis of evidence (Data Supplements 1 and 2) published between March 2006 and January 2013 to determine whether the recommendations needed to be updated. The Update Panel drafted the guideline manuscript and submitted it for review. The ASCO Clinical Practice Guideline Committee then reviewed and approved the Updated Guideline.

Details of the literature search strategy are provided in Data Supplement 3. In brief, articles were selected for inclusion in the systematic review of the evidence if they met the following criteria: (1) The study discussed a fertility intervention and reported primary data, and (2) the study population consisted of patients with cancer scheduled for or undergoing cancer treatments that threaten fertility. Articles were excluded from further consideration if they did not report specifically on a fertility intervention and did not report primary data. However, because of the limited nature of the data in many areas, the Update Panel made an a priori decision to also retain high-quality reviews or background articles. A QUOROM diagram that reports the results of the literature search is available in Data Supplement 4.

\section{Guideline Policy}

The practice guideline is not intended to substitute for the independent professional judgment of the treating physician. Practice guidelines do not account for individual variation among patients and may not reflect the most recent evidence. This guideline does not recommend any particular product or course of medical treatment. Use of the clinical practice guideline is voluntary.

\section{Guideline and Conflicts of Interest}

The Update Panel was assembled in accordance with the ASCO Conflicts of Interest Management Procedures for Clinical Practice Guidelines (Procedures, summarized at http://www.asco.org/guidelinescoi). Members of the Update Panel completed a disclosure form, which requires disclosure of financial and other interests that are relevant to the subject matter of the guideline, including relationships with commercial entities that are reasonably likely to experience direct regulatory or commercial impact as the result of promulgation of the guideline. Categories for disclosure include employment relationships, consulting arrangements, stock ownership, honoraria, research funding, and expert testimony. In accordance with the Procedures, the majority of the members of the Update Panel did not disclose any such relationships.

\section{GUIDELINE RECOMMENDATIONS}

After review and analysis of the evidence published since the original guideline appeared in Journal of Clinical Oncology in 2006, the Update Panel concluded that new evidence was not compelling enough to warrant substantive changes to any of the 2006 guideline recommendations. There were minor but significant changes worthy of attention; however, they did not necessitate a major revision of the guideline. Table 1 provides a summary of the 2013 guideline recommendations.

\section{Literature Search Results}

There were 18 new randomized controlled trials, ${ }^{2-20}$ six systematic reviews, meta-analyses, or previous guidelines, ${ }^{21-26}$ and dozens of narrative reviews, case series and case studies, and editorials. Evidence tables are presented in Data Supplements 1 and 2.

\section{Limitations of the Literature and Future Research}

Review of the fertility preservation literature revealed a paucity of large and/or randomized studies. Most data came from cohort studies, case series, small nonrandomized clinical trials, or case reports. Fertility preservation methods are still applied relatively infrequently in patients with cancer, limiting greater knowledge about the success and effects of different interventions and the long-term health of offspring. Insufficient attention is paid to the potential positive and negative effects, both physical and psychological, of fertility preservation. There is a need for research about decision making regarding the future use of cryopreserved tissue and posthumous reproduction.

Although there is current evidence that indicates a lack of effectiveness of hormonal suppression in fertility preservation, there is a need for a decisive study in which a large number of patients undergo follow-up involving sensitive ovarian reserve markers such as antiMüllerian hormone and antral follicle counts as well as, if feasible, ovarian follicle counts assessed by histologic analysis of ovaries or by xenograft models with and without gonadotropin-releasing hormone agonist and antagonist ( $\mathrm{GnRHa}$ ) treatment during chemotherapy. The penultimate study should also have sufficient power and follow-up to compare pregnancy outcomes. Thus, the Update Panel encourages participation in clinical trials that meet these criteria as long as the patients also consider alternative and effective methods of fertility preservation.

In addition, little is known about the emotional impact of infertility or the use of fertility preservation options for people with cancer in ethnically, racially, or socioeconomically diverse groups, who may face even greater barriers to fertility preservation before treatment.

The Update Panel encourages additional well-designed studies evaluating methods of fertility preservation in people with cancer to help answer these questions. Research is also needed on the comparative effectiveness of different modes of fertility preservation. However, the Panel also acknowledges that the traditional gold standard of randomized, controlled, and blinded therapeutic studies may not be practical is in this area. ASCO believes that cancer clinical trials are vital to inform medical decisions and improve cancer care, and that all patients should have the opportunity to participate.

\section{UPDATE}

This guideline update provides a brief review of key new studies under each clinical question addressing fertility preservation in adults and 
Table 1. ASCO 2013 Recommendations for Fertility Preservation for Patients With Cancer

\section{Clinical Question}

1. Are patients with cancer interested in interventions to preserve fertility?

What can health care providers do to educate patients about the possibility of reduced fertility resulting from cancer treatments and to introduce them to methods to preserve fertility?

2. What is the quality of evidence supporting current and forthcoming options for preservation of fertility in males?

3. What is the quality of evidence supporting current and forthcoming options for preservation of fertility in females?
Recommendation

1.1 People with cancer are interested in discussing fertility preservation. Health care providers caring for adult and pediatric patients with cancer (including medical oncologists, radiation oncologists, gynecologic oncologists, urologists, hematologists, pediatric oncologists, surgeons, and others) should address the possibility of infertility as early as possible before treatment starts.

1.2 Health care providers should refer patients who express an interest in fertility preservation (and patients who are ambivalent) to reproductive specialists.

1.3 Fertility preservation is often possible, but to preserve the full range of options, fertility preservation approaches should be discussed as early as possible, before treatment starts. The discussion can ultimately reduce distress and improve quality of life. Another discussion and/or referral may be necessary when the patient returns for follow-up and if pregnancy is being considered. The discussions should be documented in the medical record.

2.1 Sperm cryopreservation: Sperm cryopreservation is effective, and health care providers should discuss sperm banking with postpubertal males receiving cancer treatment.

2.2 Hormonal gonadoprotection: Hormonal therapy in men is not successful in preserving fertility. It is not recommended.

2.3 Other methods to preserve male fertility: Other methods, such as testicular tissue cryopreservation and reimplantation or grafting of human testicular tissue, should be performed only as part of clinical trials or approved experimental protocols

2.4 Postchemotherapy: Men should be advised of a potentially higher risk of genetic damage in sperm collected after initiation of therapy.

It is strongly recommended that sperm be collected before initiation of treatment because the quality of the sample and sperm DNA integrity may be compromised after a single treatment session. Although sperm counts and quality of sperm may be diminished even before initiation of therapy, and even if there may be a need to initiate chemotherapy quickly such that there may be limited time to obtain optimal numbers of ejaculate specimens, these concerns should not dissuade patients from banking sperm. Intracytoplasmic sperm injection allows the future use of a very limited amount of sperm; thus, even in these compromised scenarios, fertility may still be preserved.

3.1 Embryo cryopreservation: Embryo cryopreservation is an established fertility preservation method, and it has routinely been used for storing surplus embryos after in vitro fertilization.

3.2 Cryopreservation of unfertilized oocytes: Cryopreservation of unfertilized oocytes is an option, particularly for patients who do not have a male partner, do not wish to use donor sperm, or have religious or ethical objections to embryo freezing.

Oocyte cryopreservation should be performed in centers with the necessary expertise. As of October 2012 , the American Society for Reproductive Medicine no longer deems this procedure experimental.

More flexible ovarian stimulation protocols for oocyte collection are now available. Timing of this procedure no longer depends on the menstrual cycle in most cases, and stimulation can be initiated with less delay compared with old protocols. Thus, oocyte harvesting for the purpose of oocyte or embryo cryopreservation is now possible on a cycle day-independent schedule.

3.3 Ovarian transposition: Ovarian transposition (oophoropexy) can be offered when pelvic irradiation is performed as cancer treatment. However, because of radiation scatter, ovaries are not always protected, and patients should be aware that this technique is not always successful.

Because of the risk of remigration of the ovaries, this procedure should be performed as close to the time of radiation treatment as possible.

3.4 Conservative gynecologic surgery: It has been suggested that radical trachelectomy (surgical removal of the uterine cervix) should be restricted to stage IA2 to IB cervical cancer with diameter $<2 \mathrm{~cm}$ and invasion $<10$ $\mathrm{mm}$.

In the treatment of other gynecologic malignancies, interventions to spare fertility have generally centered on doing less radical surgery with the intent of sparing the reproductive organs as much as possible. Ovarian cystectomy can be performed for early-stage ovarian cancer.

3.5 Ovarian suppression: Currently, there is insufficient evidence regarding the effectiveness of $\mathrm{GnRHa}$ and other means of ovarian suppression in fertility preservation.

$\mathrm{GnRHa}$ should not be relied upon as a fertility preservation method. However, GnRHa may have other medical benefits such as a reduction of vaginal bleeding when patients have low platelet counts as a result of chemotherapy. This benefit must be weighed against other possible risks such as bone loss, hot flashes, and potential interference with response to chemotherapy in estrogen-sensitive cancers. Women interested in this method should participate in clinical trials, because current data do not support it. In a true emergency or rare or extreme circumstances where proven options are not available, providers may consider GnRHa an option, preferably as part of a clinical trial.

3.6 Ovarian tissue cryopreservation and transplantation: Ovarian tissue cryopreservation for the purpose of future transplantation does not require ovarian stimulation or sexual maturity and hence may be the only method available in children. It is considered experimental and should be performed only in centers with the necessary expertise, under IRB-approved protocols that include follow-up for recurrent cancer. A theoretic concern with reimplanting ovarian tissue is the potential for reintroducing cancer cells depending on the type and stage of cancer, although so far there have been no reports of cancer recurrence.

3.7 Other considerations: Of special concern in estrogen-sensitive breast and gynecologic malignancies is the possibility that fertility preservation interventions (eg, ovarian stimulation regimens that increase estrogen levels) and/or subsequent pregnancy may increase the risk of cancer recurrence.

Ovarian stimulation protocols using the aromatase inhibitor letrozole have been developed and may ameliorate this concern. Studies do not indicate increased cancer recurrence risk as a result of subsequent pregnancy. (continued on following page) 
Table 1. ASCO 2013 Recommendations for Fertility Preservation for Patients With Cancer (continued)

\begin{tabular}{|c|c|}
\hline Clinical Question & Recommendation \\
\hline $\begin{array}{l}\text { 4. What is the role of health care } \\
\text { providers in advising patients about } \\
\text { fertility preservation options? }\end{array}$ & $\begin{array}{l}\text { 4.1 All oncologic health care providers should be prepared to discuss infertility as a potential risk of therapy. This } \\
\text { discussion should take place as soon as possible once a cancer diagnosis is made and before a treatment } \\
\text { plan is formulated. There are benefits for patients in discussing fertility information with providers at every } \\
\text { step of the cancer journey. }\end{array}$ \\
\hline \multirow{3}{*}{$\begin{array}{l}\text { What should providers discuss with } \\
\text { patients about fertility } \\
\text { preservation? }\end{array}$} & $\begin{array}{l}\text { 4.2 Encourage patients to participate in registries and clinical studies, as available, to define further the safety and } \\
\text { efficacy of these interventions and strategies }\end{array}$ \\
\hline & $\begin{array}{l}\text { 4.3 Refer patients who express an interest in fertility, as well as those who are ambivalent or uncertain, to } \\
\text { reproductive specialists as soon as possible. }\end{array}$ \\
\hline & 4.4 Refer patients to psychosocial providers when they are distressed about potential infertility. \\
\hline \multirow[t]{2}{*}{$\begin{array}{l}\text { Special considerations: Fertility } \\
\text { preservation in children }\end{array}$} & $\begin{array}{l}\text { 5.1 Suggest established methods of fertility preservation (eg, semen or oocyte cryopreservation) for postpubertal } \\
\text { minor children, with patient assent and parent or guardian consent. }\end{array}$ \\
\hline & $\begin{array}{l}\text { For prepubertal minor children, the only fertility preservation options are ovarian and testicular } \\
\text { cryopreservation, which are investigational. }\end{array}$ \\
\hline
\end{tabular}

Abbreviations: ASCO, American Society of Clinical Oncology; GnRHa, gonadotropin-releasing hormone analog; IRB, institutional review board.

children undergoing treatment for cancer. The language has been clarified and/or strengthened in several recommendations. Information has been added to address role of psychosocial providers, fertility preservation concerns, and options for children and adolescents with cancer, as well as considerations for patients receiving targeted and biologic therapies in this update.

After a systematic review and analysis of the literature for the preservation of fertility for patients with cancer, the Update Panel concluded that there was no new evidence compelling enough to warrant substantial changes to any of the guideline recommendations. However, minor adjustments were made to reflect progress in the field (eg, oocyte cyropreservation is no longer investigational). Certainly, further research is needed to determine the true effectiveness of different modes of fertility preservation. More research is also needed to establish the best methods to disseminate information and to determine the best time to talk with patients about their options. The discussion should be a part of the comprehensive treatment planning process (Fig 1). The treatment planning discussion should include consideration of scientific evidence, weighing potential harms and benefits, reproductive potential, anticipated delay of childbearing, and patient preferences. The Update Panel strongly encourages health care providers to have an open dialogue with patients or parents or guardians of children anticipating cancer treatment who express an interest in fertility preservation (and those patients who are ambivalent) and refer them as expeditiously as possible to a reproductive specialist, preferably before starting treatment. Electronic resources (eg, e-mail, Skype) are available that may facilitate novel methods of consultation, such as telephone- or Internet-based communication, for patients without geographic accessibility to these specialized providers.

\section{Are Patients With Cancer Interested in Interventions to Preserve Fertility?}

Current evidence suggests that discussions about fertility and fertility preservation are of great importance to patients with cancer. ${ }^{27}$ It may be difficult for physicians to know how important fertility preservation is to their patients unless they ask, because many patients may not bring up the topic. The failure of patients to mention infertility concerns or interest in fertility preservation can result from a variety of factors; they may be overwhelmed by and focused exclusively on the cancer diagnosis, ${ }^{28}$ they may be unaware that potential fertility loss may occur, ${ }^{29}$ or they may be concerned that pursuing

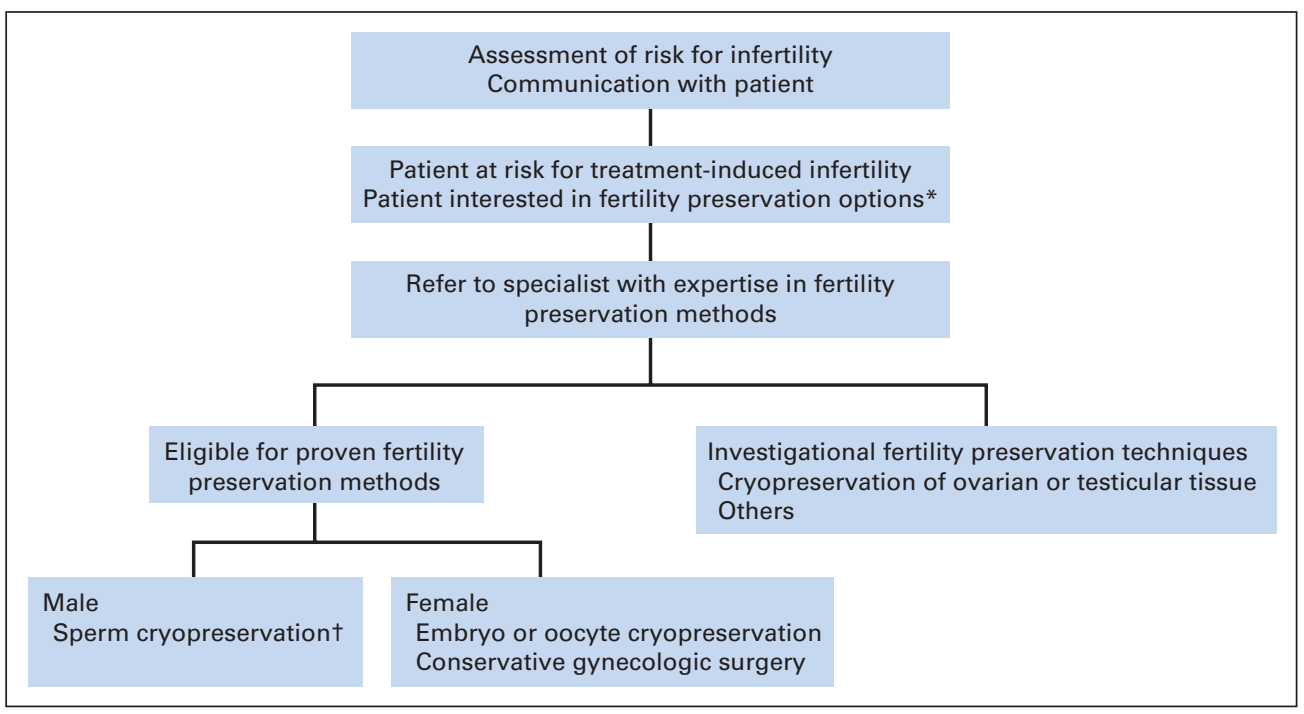

Fig 1. Fertility preservation assessment and discussion algorithm for patients with cancer. ${ }^{*}$ ) Patients should be encouraged to contact their insurance company to ascertain out-of-pocket costs. ( $t$ ) Some patients may proceed with this without the prior step of seeing a reproductive specialist. However, consultation with a reproductive specialist is recommended. 
fertility preservation will delay their treatment, leading to increased morbidity or mortality. ${ }^{30}$

However, there is evidence to suggest that at least among women, patients may make cancer treatment decisions based on fertility concerns. In the study by Partridge et al, ${ }^{31} 29 \%$ of women with breast cancer reported that infertility concerns influenced their treatment decisions.

\section{What Is the Quality of Evidence Supporting Current and Forthcoming Options for Preservation of Fertility in Males?}

The treatment of cancer often poses a threat to male fertility. Understanding the effects of different antitumor agents on sperm production in men has changed little in the 7 years since the original guideline was published. An updated table on the effects of different antitumor agents on sperm production and a summary of fertility preservation options in males are presented in Data Supplement 5.

The Panel reviewed recent information supporting sperm cryopreservation, testicular hormonal suppression, and testicular tissue cryopreservation. The new evidence continues to support the conclusion that sperm cryopreservation is an effective method of fertility preservation in males treated for cancer. ${ }^{32-39}$ In contrast, gonadoprotection through hormonal manipulation is ineffective. Testicular tissue or spermatogonial cryopreservation and transplantation or testis xenografting are still experimental and have not yet been successfully tested in humans. However, such approaches may be the only methods of fertility preservation potentially available to prepubertal boys. There are case reports and small case series of successful collection of sperm from a postmasturbation urine sample, rectal electroejaculation under anesthesia, and testicular sperm aspiration, but these remain uncommon and/or investigational. It also seems that testicular cryopreservation procedures can be combined with other medically indicated procedures to increase the feasibility and acceptability of these procedures. ${ }^{40}$ The Update Panel notes that if patients are promptly referred to a fertility specialist, there is likely to be little to no significant delay in the initiation of cancer treatment.

\section{What Is the Quality of Evidence Supporting Current and Forthcoming Options for Preservation of Fertility in Females?}

Understanding of the risks of permanent amenorrhea in women treated with modern chemotherapy and radiotherapy has changed little since the original guideline. However, there have been some advances in the science of fertility preservation that may affect patient decision making. An updated table on the risks of permanent amenorrhea in women treated with modern chemotherapy and radiotherapy and a summary of fertility preservation options in females is presented in Data Supplement 6.

The Panel reviewed the new literature supporting embryo and oocyte cryopreservation (with hormonal stimulation), ovarian transposition, surgical options other than radical trachelectomy, ovarian suppression, ovarian tissue cryopreservation and transplantation, and other considerations. Fertility preservation options in females depend on patient age, diagnosis, type of treatment, presence or participation of a male partner and/or patient preferences regarding the use of banked donor sperm, time available, and likelihood that cancer has metastasized to her ovaries. The Update Panel notes that because of requirements for scheduling and performing procedures, some (but not all) interventions may entail a delay in cancer treatment and wishes to emphasize that early referral to a subspecialist can minimize this delay.

Embryo cryopreservation. New data indicate that although it is ideal to stimulate ovaries within 3 days of the start of the menstrual cycle, random stimulation can be successful as well. ${ }^{41}$ This is an important and recent advance in the field of reproductive endocrinology. Furthermore, newer hormonal stimulation regimens (eg, letrozole and tamoxifen) may be effective as traditional methods, and their use may be preferred in women with hormone-sensitive cancers. $3,6,12,42,43$

Although aromatase inhibitors are primarily used as adjuvant treatment of hormone-positive breast cancers (in premenopausal women), they can act as ovarian stimulants yet suppress estrogen levels. As a result, letrozole has been used for ovulation induction in infertility patients and, in the last 10 years, for the purpose of ovarian stimulation for fertility preservation via oocyte or embryo cryopreservation in women with estrogen-sensitive cancer. When combined with standard fertility drugs, letrozole enhances ovarian stimulation while keeping estrogen levels near physiologic levels. Studies suggest that this approach results in similar numbers of eggs and embryos and similar pregnancy outcomes. Short-term follow-up indicated no impact on cancer-free survival. The Update Panel wishes to emphasize these developments because they may widen the opportunities for fertility preservation.

Cryopreservation of unfertilized oocytes. Success rates for this procedure have improved significantly, and it is no longer considered experimental by the American Society of Reproductive Medicine. Some reproductive specialty centers have reported success rates comparable to those obtained using unfrozen eggs, especially in younger women. ${ }^{9,44-46}$ Like embryo cryopreservation, this technique also requires ovarian stimulation and ultrasound-guided oocyte retrieval. Oocyte cryopreservation is of particular importance for women who do not have a male partner or prefer not to use donor sperm.

Ovarian suppression. The question regarding the effectiveness of $\mathrm{GnRHa}$ is still not resolved. One recent study with flaws ${ }^{2}$ cited a slight benefit for return of menstruation, but another article ${ }^{47}$ showed no significant difference in the outcome point of chemotherapy-induced amenorrhea 6 months after the end of chemotherapy. A recent study demonstrated no benefit of using GnRHa in patients with breast cancer receiving cyclophosphamide-based chemotherapy. ${ }^{48}$ In this study, no differences were observed in the menstruation resumption rates between GnRHa-treated patients versus the control group 12 months after termination of chemotherapy. Moreover, there were no differences in hormonal and ultrasound markers of fertility between patients receiving GnRHa and the control group. The use of GnRHa cotreatment did not predict independently the odds of menstruating at 12 months. Furthermore, a recent meta-analysis, which updates an earlier one, included 24 months of follow-up in the ZORO (Zoladex Rescue of Ovarian Function) study $^{49}$ and failed to demonstrate a possible beneficial effect of GnRHa use on either maintenance of menstruation or fertility. There are not definitive data ${ }^{5,11,21,50}$ that show that GnRHa preserves fertility, and it remains the subject of ongoing research.

Given the current state of knowledge regarding these agents, it is the opinion of the Update Panel that GnRHa is not an effective method of fertility preservation. Furthermore, complete ovarian suppression is not achieved for several weeks after administration. However, there may be other potential benefits such as inhibiting menses 
during intensive chemotherapy, thus preventing complications such as menorrhagia. In emergency, rare, or extreme circumstances, where proven options are not available, providers may consider GnRHa an unproven option (preferably as a part of a clinical trial), with special consideration of the patient's specific cancer and needs. This class of drugs also has adverse effects such as hot flashes and bone loss.

Ovarian tissue cryopreservation and transplantation. Although this process is still considered experimental, successful pregnancies have been reported. There is a theoretic concern with reimplanting ovarian tissue and the potential for reintroducing cancer cells depending on the type and stage of cancer, although so far there have been no reports of cancer recurrence in humans. In women who have survived cancer, at least 19 live births have been reported using cryopreserved ovarian tissue or oocytes. ${ }^{7,19,51-58}$

Other considerations. (1) With recent data supporting longer duration of hormonal therapies for estrogen receptor/progesterone receptor-positive breast cancer, larger numbers of women will be affected by the risk of compromised fertility. ${ }^{42}$ These women will be older and thus at higher risk for infertility at the time that their hormonal therapy is completed. (2) It has been shown that BRCA mutation carriers, especially those with $B R C A 1$, have diminished ovarian reserve. ${ }^{59}$ There is a concern that $B R C A$ mutation carriers may be more prone to chemotherapy-induced infertility as a result of already lower ovarian reserve and higher likelihood of low response to ovulation induction. This may be important when counseling women regarding their likelihood of infertility after chemotherapy. (3) For patients with inherited or familial cancers for which a mutation has been identified, there may be an added benefit of undergoing fertility preservation by oocyte or embryo cryopreservation, because embryos can be tested for these mutations by embryo biopsy, and preimplantation genetic diagnosis techniques can be considered. (4) A number of conservative surgical (eg, trachelectomy $)^{60-64}$ and radiation therapy approaches with the aim of preserving fertility are available but are not discussed further in this guideline. Surgical and radiation oncologists should discuss individualized approaches with specific patients, taking into account patient preferences, risks, specific tumor anatomy, and other concerns.

\section{What Is the Role of the Health Care Provider in Advising Patients About Fertility Preservation Options? What Should Providers Discuss With Patients About Fertility Preservation?}

As with other potential complications of cancer treatment, all health care providers have a responsibility to inform patients about the risks that their cancer treatment will permanently impair fertility. Providers should encourage patients to look into insurance coverage (state-by-state differences) and out-of-pocket costs (which may be supported by charitable funding). An algorithm for triaging fertility preservation referrals is presented in Figure 1.

There are many new studies addressing the importance and timing of referral to reproductive specialists and psychosocial providers. Referrals should be made as soon as possible. Psychosocial providers such as social workers and psychologists can be particularly helpful when a patient is distressed about potential infertility. Some patients, after successful cancer treatment, may want to have a biologic child. The inability to conceive could be a great source of distress. Although it is ideal for a patient to discuss threats to fertility and potential options before cancer treatment, there are other family building options that can be used postcancer. These include the use of gestational carriers, embryo donation, egg or sperm donation, and adoption. Psychosocial providers can assist patients and families in the decision-making process about fertility preservation and disposition of stored gamete options that are morally and ethically acceptable to them. ${ }^{29,34,65-87}$

Fertility preservation does not diminish the chance of successful cancer treatment. However, if a patient received a treatment that affects cardiopulmonary function, she should be evaluated by an appropriate specialist (eg, maternal-fetal medicine, cardiology, or pulmonology) before attempting pregnancy. If a woman underwent pelvic irradiation, this should be discussed with a maternalfetal medicine specialist as well, because pregnancy complications such as intrauterine growth retardation and preterm delivery may occur as a result of uterine dysfunction.

\section{Special Fertility Preservation Considerations for Children and Adolescents With Cancer}

There are new observational studies, as well as case studies, addressing fertility preservation of children and adolescents with cancer, including the risks of radiation as well as chemotherapy. ${ }^{88-92}$ Parents or guardians are often interested in information about fertility preservation on behalf of their children with cancer. Impaired future fertility is difficult for children to understand but potentially may be traumatic to them as adults. Use of established methods of fertility preservation (eg, semen cryopreservation and oocyte freezing) in postpubertal minor children requires patient assent and parental consent. Unfortunately, there are no standard modalities available for fertility preservation in prepubertal children. Current techniques are limited by the patient's sexual immaturity, and all available approaches for children are experimental. Ooctye cryopreservation has been reported in children age 1 years and older. There have been numerous reports of ovarian cryopreservation in younger children, also, but there have been no reports of live births after ovarian cortical tissue cryopreserved prepubertally and reimplanted at a later date, primarily because of the young age of the study participants. ${ }^{93,94}$ Efforts to preserve fertility of children using experimental methods should be attempted only under institutional review board-approved protocols. Likewise, testicular cryopreservation has used in young children, but there are no reports of testicular transplantation in the peer-reviewed literature.

Several studies confirm that adult survivors of pediatric cancer wish they had been given more information and options about fertility, and these survivors are often uncertain about their fertility status or feel regret about no longer having an option. ${ }^{95,96}$ Parents may be uncertain about making fertility related decisions on behalf of a minor; both the American Academy of Pediatrics ${ }^{97}$ and the American Society for Reproductive Medicine ${ }^{25}$ offer guidance for counseling parents of children with cancer.

\section{Special Fertility Preservation Considerations for Patients Receiving Targeted and Biologic Therapies}

Since the publication of the 2006 guidelines, the number of novel agents and classes of therapeutic agents has expanded significantly. The Panel acknowledges that there is little available information regarding the impact of these agents on fertility, at any level of evidence, for the vast majority of these treatment modalities. One important exception is bevacizumab, for which the US Food and Drug Administration issued a warning in October 2011, reporting that ovarian 
failure occurred in 34\% of women receiving a bevacizumabcontaining regimen for colorectal cancer compared with $2 \%$ of women receiving the same regimen without bevacizumab. Only approximately one fifth of these women recovered ovarian function. The US Food and Drug Administration therefore recommends that oncologists "inform females of reproductive potential of the risk of ovarian failure before starting treatment with bevacizumab." ${ }^{98}$ Another specific area of concern frequently encountered by clinicians is how to counsel young patients with chronic myeloid leukemia in chronic phase who are being managed with tyrosine kinase inhibitors (TKIs) such as imatinib. Although recommendations regarding management of these patients is beyond the scope of this guideline, the Update Panel wishes to note that a number of case reports, case series, and expert reviews have been published suggesting that young men receiving TKIs probably do not confer an increased risk of pregnancyrelated complications or congenital anomalies to their partners and offspring, ${ }^{99}$ although these men should be counseled and strongly cautioned that there are insufficient data to provide adequate reassurance. Similar level of evidence suggests that women of reproductive capacity should not become pregnant while taking TKIs, because there is strong evidence that these agents are teratogenic in animal models.

\section{PROVIDER AND PATIENT COMMUNICATION}

Health care providers can use the following points for a discussion of infertility and fertility preservation with a patient (or parents or guardians):

Inform patient of individual risk

- Some cancer treatments can cause infertility or early menopause.

- To determine your individual risks, we have considered your individual factors such as your cancer type, age, and treatment plan.

- Based on that information, we believe that your risk is [high, medium, low, nonexistent].

- Your fertility status before cancer may also play a role in your individual risks [discuss if relevant].

Discuss common concerns

Options

- There are many available fertility preservation and parenthood after cancer options for you to consider.

- For men, the most common and successful option is sperm banking. Other experimental options exist, if sperm banking is not a viable option for you.

- For women, the most established options are embryo and egg freezing. Other experimental options exist, if these are not viable options for you.

- A referral can be made for you to an appropriate reproductive specialist for a consultation, if you would like to learn more.

Time

- Time is of the essence. Fertility preservation treatments need to be completed before you start chemotherapy and/ or irradiation.

- For men, sperm banking can be done quickly and can be done every 24 hours, as long as necessary, to collect the desired number of samples.

- For women, fertility preservation may take 2 to 4 weeks for established techniques. However, some experimental approaches can implemented sooner, so timely referral to a reproductive specialist is important.

Costs

- Insurance coverage for fertility preservation for patients with cancer is improving. The fertility center/sperm bank will be able to check your benefits for you.

- Advocacy organizations such as LIVESTRONG Fertile Hope and some pharmaceutical companies may also provide costsaving programs.

Risks of pregnancy and children after cancer

- Many patients worry about the safety of pregnancy after cancer. Data are limited, but there seems to be no increased risk of cancer recurrence from fertility preservation methods or pregnancy, even in hormonally sensitive tumors.

- Similarly, many patients worry about the risk of passing cancer along to their children. Aside from hereditary genetic syndromes and in utero exposure to some chemotherapy treatments, there is no evidence that a history of cancer, cancer therapy, or fertility interventions increases the risk of cancer or congenital abnormalities in the progeny.

Refer to appropriate specialists

- Reproductive specialists: For more information about fertility preservation, a referral can be provided you to a local fertility specialist/sperm bank.

- Mental health professionals: Many patients find cancer treatmentrelated infertility distressing. There is a lot to think about in addition to cancer. You can be referred to a counselor, if that would be helpful. Many of the reproductive centers also have counselors available to discuss these issues, so you may be able to see someone while there for your consultation.

- Advocacy organizations: Many advocacy organizations such as LIVESTRONG Foundation's Fertile Hope Program and the Oncofertility Consortium also provide useful information and resources to help facilitate your decision making. They may also have financial assistance programs specifically designed to help with fertility preservation.

\section{HEALTH DISPARITIES}

Although ASCO clinical practice guidelines represent expert recommendations on the best practices in disease management to provide the highest level of cancer care, it is important to note that many patients have limited access to medical care. Racial, ethnic, and socioeconomic disparities in health care contribute significantly to this problem in the United States. Minority racial/ethnic patients with cancer suffer disproportionately from comorbidities, can experience substantial obstacles to receiving care, are more likely to be uninsured, and are at greater risk of receiving poorer quality care than other Americans. ${ }^{100-103}$ Many other patients lack access to care because they live at a distance from appropriate treatment or reproductive specialty facilities.

Awareness of these disparities in access to care should be considered in the context of this clinical practice guideline, and health care providers should strive to deliver the highest-level fertility preservation advice and treatment to these vulnerable populations. In particular, no patient should be excluded from consideration for discussion 
of fertility preservation for any reason, including age, prognosis, socioeconomic status, or parity. In discussion, all patients including parents or guardians of children and adolescents should be encouraged to consider fertility preservation, even though there may be financial or insurance barriers. Discussing infertility and introducing the possibility of fertility preservation leads to improved quality of life and diminished distress in all patient populations.

\section{ADDITIONAL RESOURCES}

Data Supplements and clinical tools and resources can be found at http://www.asco.org/guidelines/fertility. Patient information is also available at http://www.cancer.net.

\section{AUTHORS' DISCLOSURES OF POTENTIAL CONFLICTS} OF INTEREST

The author(s) indicated no potential conflicts of interest.

\section{AUTHOR CONTRIBUTIONS}

\author{
Administrative support: Alison W. Loren, Pamela B. Mangu, \\ Kutluk Oktay \\ Provision of study materials or patients: Kutluk Oktay \\ Manuscript writing: All authors \\ Final approval of manuscript: All authors
}

\section{REFERENCES}

1. Lee SJ, Schover LR, Partridge $A H$, et al: American Society of Clinical Oncology recommendations on fertility preservation in cancer patients. J Clin Oncol 24:2917-2931, 2006

2. Del Mastro $L$, Boni $L$, Michelotti $A$, et al: Effect of the gonadotropin-releasing hormone analogue triptorelin on the occurrence of chemotherapy-induced early menopause in premenopausal women with breast cancer: A randomized trial. JAMA 306:269-276, 2011

3. Azim AA, Costantini-Ferrando M, Oktay K: Safety of fertility preservation by ovarian stimulation with letrozole and gonadotropins in patients with breast cancer: A prospective controlled study. J Clin Oncol 26:2630-2635, 2008

4. Munster PN, Moore AP, Ismail-Khan R, et al: Randomized trial using gonadotropin-releasing hormone agonist triptorelin for the preservation of ovarian function during (neo)adjuvant chemotherapy for breast cancer. J Clin Oncol 30:533-538, 2012

5. Behringer $K$, Wildt L, Mueller $H$, et al: No protection of the ovarian follicle pool with the use of $\mathrm{GnRH}$-analogues or oral contraceptives in young women treated with escalated BEACOPP for advanced-stage Hodgkin lymphoma: Final results of a phase II trial from the German Hodgkin Study Group. Ann Oncol 21:2052-2060, 2010

6. Oktay K, Buyuk E, Libertella N, et al: Fertility preservation in breast cancer patients: A prospective controlled comparison of ovarian stimulation with tamoxifen and letrozole for embryo cryopreservation. J Clin Oncol 23:4347-4353, 2005

7. Barton SE, Missmer SA, Berry KF, et al: Female cancer survivors are low responders and have reduced success compared with other patients undergoing assisted reproductive technologies. Fertil Steril 97:381-386, 2012

8. Behringer $\mathrm{K}$, Thielen $\mathrm{I}$, Mueller $\mathrm{H}$, et al: Fertility and gonadal function in female survivors after treatment of early unfavorable Hodgkin lymphoma (HL) within the German Hodgkin Study Group HD14 trial. Ann Oncol 23:1818-1825, 2012

9. Bacigalupo A, Ballen K, Rizzo D, et al: Defining the intensity of conditioning regimens: Working definitions. Biol Blood Marrow Transplant 15:16281633, 2009

10. de Lavallade $H$, Finetti $P$, Carbuccia $N$, et al: A gene expression signature of primary resistance to imatinib in chronic myeloid leukemia. Leuk Res 34:254-257, 2010

11. Oktay K, Türkçüoğlu I, Rodriguez-Wallberg $\mathrm{KA}$ : GnRH agonist trigger for women with breast cancer undergoing fertility preservation by aromatase inhibitor/FSH stimulation. Reprod Biomed Online 20:783-788, 2010

12. Lee S, Oktay K: Does higher starting dose of FSH stimulation with letrozole improve fertility preservation outcomes in women with breast cancer? Fertil Steril 98:961.e1-964.e1, 2012

13. Das $M$, Shehata F, Son WY, et al: Ovarian reserve and response to IVF and in vitro maturation treatment following chemotherapy. Hum Reprod 27:2509-2514, 2012

14. van Hanegem N, Barroilhet LM, Nucci MR, et al: Fertility-sparing treatment in younger women with adenocarcinoma in situ of the cervix. Gynecol Oncol 124:72-77, 2012

15. Nicolini FE, Mauro MJ, Martinelli G, et al: Epidemiologic study on survival of chronic myeloid leukemia and $\mathrm{Ph}(+)$ acute lymphoblastic leukemia patients with BCR-ABL T315I mutation. Blood 114: 5271-5278, 2009

16. Almog B, Azem F, Gordon D, et al: Effects of cancer on ovarian response in controlled ovarian stimulation for fertility preservation. Fertil Steril 98: 957-960, 2012

17. Han SS, Kim YH, Lee SH, et al: Underuse of ovarian transposition in reproductive-aged cancer patients treated by primary or adjuvant pelvic irradiation. J Obstet Gynaecol Res 37:825-829, 2011

18. Terenziani M, Piva L, Meazza C, et al: Oophoropexy: A relevant role in preservation of ovarian function after pelvic irradiation. Fertil Steril 91: 935.e15-935.e16, 2009

19. Halter J, Kodera Y, Ispizua AU, et al: Severe events in donors after allogeneic hematopoietic stem cell donation. Haematologica 94:94-101, 2009

20. Checa Vizcaíno MA, Corchado AR, Cuadri $M E$, et al: The effects of letrozole on ovarian stimulation for fertility preservation in cancer-affected women. Reprod Biomed Online 24:606-610, 2012

21. Clare S, Mank A, Stone R, et al: Management of related donor care: A European survey. Bone Marrow Transplant 45:97-101, 2010

22. Detti L, Martin DC, Williams LJ: Applicability of adult techniques for ovarian preservation to childhood cancer patients. J Assist Reprod Genet 29: 985-995, 2012

23. Pentheroudakis G, Pavlidis N, Castiglione M: Cancer, fertility and pregnancy: ESMO clinical recommendations for diagnosis, treatment and followup. Ann Oncol 20:178-181, 2009 (suppl 4)

24. Donnez J, Dolmans MM: Cryopreservation and transplantation of ovarian tissue. Clin Obstet Gynecol 53:787-796, 2010

25. American Society for Reproductive Medicine. http://www.asrm.org
26. Bedaiwy MA, Abou-Setta AM, Desai N, et al: Gonadotropin-releasing hormone analog cotreatment for preservation of ovarian function during gonadotoxic chemotherapy: A systematic review and meta-analysis. Fertil Steril 95:906-914, e1-e4, 2011

27. Maltaris $T$, Seufert R, Fischl F, et al: The effect of cancer treatment on female fertility and strategies for preserving fertility. Eur J Obstet Gynecol Reprod Biol 130:148-155, 2007

28. Klock SC, Zhang JX, Kazer RR: Fertility preservation for female cancer patients: Early clinical experience. Fertil Steril 94:149-155, 2010

29. Schover LR, Brey K, Lichtin A, et al: Oncologists' attitudes and practices regarding banking sperm before cancer treatment. J Clin Oncol 20: 1890-1897, 2002

30. Achille MA, Rosberger $Z$, Robitaille $R$, et al: Facilitators and obstacles to sperm banking in young men receiving gonadotoxic chemotherapy for cancer: The perspective of survivors and health care professionals. Hum Reprod 21:3206-3216, 2006

31. Partridge AH, Gelber $S$, Peppercorn J, et al: Web-based survey of fertility issues in young women with breast cancer. J Clin Oncol 22:41744183,2004

32. Hourvitz A, Goldschlag DE, Davis OK, et al: Intracytoplasmic sperm injection (ICSI) using cryopreserved sperm from men with malignant neoplasm yields high pregnancy rates. Fertil Steril 90: 557-563, 2008

33. Romerius $\mathrm{P}$, Ståhl $\mathrm{O}$, Moëll $\mathrm{C}$, et al: Sperm DNA integrity in men treated for childhood cancer. Clin Cancer Res 16:3843-3850, 2010

34. Salonia A, Gallina A, Matloob R, et al: Is sperm banking of interest to patients with nongerm cell urological cancer before potentially fertility damaging treatments? J Urol 182:1101-1107, 2009

35. Schmidt $\mathrm{KT}$, Andersen $\mathrm{CY}$ : Recommendations for fertility preservation in patients with lymphomas. J Assist Reprod Genet 29:473-477, 2012

36. Chang HC, Chen SC, Chen J, et al: Initial 10-year experience of sperm cryopreservation services for cancer patients. J Formos Med Assoc 105:1022-1026, 2006

37. Yee S, Fuller-Thomson E, Dwyer C, et al: "Just what the doctor ordered": Factors associated with oncology patients' decision to bank sperm. Can Urol Assoc J 6:E174-E178, 2012

38. Ping $P$, Zhu $W B$, Zhang $X Z$, et al: Sperm banking for male reproductive preservation: A 6-year retrospective multi-centre study in China. Asian J Androl 12:356-362, 2010

39. Navarro Medina P, Barroso Deyne E, Castillo Suárez $M$, et al: An analysis of our experience in 
cryopreservation of semen from cancer patients [in Spanish]. Actas Urol Esp 34:101-105, 2010

40. Babayev SN, Arslan E, Kogan S, et al: Evaluation of ovarian and testicular tissue cryopreservation in children undergoing gonadotoxic therapies. J Assist Reprod Genet :3-9,

41. Sönmezer $M$, Türkçüoğlu $I$, Coşkun $U$, et al: Random-start controlled ovarian hyperstimulation for emergency fertility preservation in letrozole cycles. Fertil Steril 95:2125.e9-2125.e11, 2011

42. Oktay K, Hourvitz A, Sahin G, et al: Letrozole reduces estrogen and gonadotropin exposure in women with breast cancer undergoing ovarian stimulation before chemotherapy. J Clin Endocrinol Metab 91:3885-3890, 2006

43. Azim AA, Costantini-Ferrando M, Lostritto $K$, et al: Relative potencies of anastrozole and letrozole to suppress estradiol in breast cancer patients undergoing ovarian stimulation before in vitro fertilization. J Clin Endocrinol Metab 92:2197-2200, 2007

44. Borini A, Bianchi V: Cryopreservation of mature and immature oocytes. Clin Obstet Gynecol 53:763-774, 2010

45. Huang JY, Chian RC, Gilbert L, et al: Retrieval of immature oocytes from unstimulated ovaries followed by in vitro maturation and vitrification: A novel strategy of fertility preservation for breast cancer patients. Am J Surg 200:177-183, 2010

46. Rudick B, Opper N, Paulson R, et al: The status of oocyte cryopreservation in the United States. Fertil Steril 94:2642-2646, 2010

47. Loibl S, Gerber B: Gonadotropin-releasing hormone analogue for premenopausal women with breast cancer. JAMA 306:1760, 2011; author reply 1760-1761

48. Elgindy EA, El-Haieg DO, Khorshid OM, et al Gonadatrophin suppression to prevent chemotherapy-induced ovarian damage: A randomized controlled trial. Obstet Gynecol 121:78-86, 2013

49. Pantanowitz L, Prefontaine M, Hunt JP: Cholelithiasis of the ovary after laparoscopic cholecystectomy: A case report. J Reprod Med 52:968-970, 2007

50. Blumenfeld $Z$, von Wolff $M$ : GnRHanalogues and oral contraceptives for fertility preservation in women during chemotherapy. Hum Reprod Update 14:543-552, 2008

51. Oktay K, Cil AP, Bang H: Efficiency of oocyte cryopreservation: A meta-analysis. Fertil Steril 86 70-80, 2006

52. Donnez J, Squifflet J, Jadoul P, et al: Pregnancy and live birth after autotransplantation of frozen-thawed ovarian tissue in a patient with metastatic disease undergoing chemotherapy and hematopoietic stem cell transplantation. Fertil Steri 95:1787.e1-1787.e4, 2011

53. Kim MK, Lee DR, Han JE, et al: Live birth with vitrified-warmed oocytes of a chronic myeloid leukemia patient nine years after allogenic bone marrow transplantation. J Assist Reprod Genet 28: 1167-1170, 2011

54. Isachenko V, Isachenko E, Keck G, et al: First live birth in Germany after re-transplantation of cryopreserved ovarian tissue: Original device for initiation of ice formation. Clin Lab 58:933-938, 2012

55. Dittrich R, Lotz L, Keck G, et al: Live birth after ovarian tissue autotransplantation following overnight transportation before cryopreservation. Fertil Steril 97:387-390, 2012

56. Andersen CY, Silber SJ, Berghold SH, et al: Long-term duration of function of ovarian tissue transplants: Case reports. Reprod Biomed Online 25:128-132, 2012
57. Oktay K, Türkçüoğlu I, Rodriguez-Wallberg KA: Four spontaneous pregnancies and three live births following subcutaneous transplantation of frozen banked ovarian tissue: What is the explanation? Fertil Steril 95:804.e7-804.e10, 2011

58. Meirow D, Levron J, Eldar-Geva T, et al: Pregnancy after transplantation of cryopreserved ovarian tissue in a patient with ovarian failure after chemotherapy. N Engl J Med 353:318-321, 2005

59. Titus S, Li F, Stobezki R, et al: Impairment of BRCA1-related DNA double-strand break repair leads to ovarian aging in mice and humans. Sc Transl Med 5:172ra21, 2013

60. Robova $H$, Pluta $M$, Hrehorcak $M$, et al: High-dose density chemotherapy followed by simple trachelectomy: Full-term pregnancy. Int J Gynecol Cancer 18:1367-1371, 2008

61. Plante M, Smith EB, Cox S, et al: The case of a viable pregnancy post vaginal radical trachelectomy followed by combined chemo-radiation therapy. Gynecol Oncol 123:421-423, 2011

62. Knight LJ, Acheson N, Kay TA, et al: Obstetric management following fertility-sparing radical vaginal trachelectomy for cervical cancer. J Obstet Gynaecol 30:784-789, 2010

63. Raju SK, Papadopoulos AJ, Montalto SA, et al: Fertility-sparing surgery for early cervical cancer: Approach to less radical surgery. Int J Gynecol Cancer 22:311-317, 2012

64. Han $L$, Yang $X Y$, Zheng $A$, et al: Systematic comparison of radical vaginal trachelectomy and radical hysterectomy in the treatment of early-stage cervical cancer. Int J Gynaecol Obstet 112:149-153, 2011

65. Ruddy KJ, Gelber S, Ginsburg ES, et al Menopausal symptoms and fertility concerns in premenopausal breast cancer survivors: A comparison to age- and gravidity-matched controls. Menopause 18:105-108, 2011

66. Peate $M$, Meiser $B$, Friedlander $M$, et al: Development and pilot testing of a fertility decision aid for young women diagnosed with early breast cancer. Breast J 17:112-114, 2011

67. Peate M, Meiser B, Friedlander M, et al: It's now or never: Fertility-related knowledge, decisionmaking preferences, and treatment intentions in young women with breast cancer-An Australian fertility decision aid collaborative group study. J Clin Oncol 29:1670-1677, 2011

68. van den Berg $H$, Langeveld NE: Parental knowledge of fertility in male childhood cancer survivors. Psychooncology 17:287-291, 2008

69. Scanlon M, Blaes A, Geller M, et al: Patient satisfaction with physician discussions of treatment impact on fertility, menopause and sexual health among pre-menopausal women with cancer. J Cancer 3:217-225, 2012

70. Peddie VL, Porter M, Bhattacharya S: A fertile future? Life after cancer in young men. Expert Rev Anticancer Ther 7:1069-1073, 2007

71. Kumar A, Merali A, Pond GR, et al: Fertility risk discussions in young patients diagnosed with colorectal cancer. Curr Oncol 19:155-159, 2012

72. Balthazar U, Deal AM, Fritz MA, et al: The current fertility preservation consultation model: Are we adequately informing cancer patients of their options? Hum Reprod 27:2413-2419, 2012

73. Schover LR, Rhodes MM, Baum G, et al: Sisters Peer Counseling in Reproductive Issues After Treatment (SPIRIT): A peer counseling program to improve reproductive health among African American breast cancer survivors. Cancer 117:49834992, 2011
74. Gorman JR, Usita PM, Madlensky L, et al: Young breast cancer survivors: Their perspectives on treatment decisions and fertility concerns. Cancer Nurs 34:32-40, 2011

75. Murphy D, Sawczyn KK, Quinn GP: Using a patient-centered approach to develop a fertility preservation brochure for pediatric oncology patients: A pilot study. J Pediatr Adolesc Gynecol 25:114-121, 2012

76. Kent EE, Parry C, Montoya MJ, et al: "You're too young for this": Adolescent and young adults' perspectives on cancer survivorship. J Psychosoc Oncol 30:260-279, 2012

77. Sait $\mathrm{KH}$ : Conservative treatment of ovarian cancer: Safety, ovarian function preservation, reproductive ability, and emotional attitude of the patients in Saudi Arabia. Saudi Med J 32:913-918, 2011

78. Yee S, Abrol K, McDonald M, et al: Addressing oncofertility needs: Views of female cancer patients in fertility preservation. J Psychosoc Oncol 30:331-346, 2012

79. de Vries MC, Bresters D, Engberts DP, et al: Attitudes of physicians and parents towards discussing infertility risks and semen cryopreservation with male adolescents diagnosed with cancer. Pediatr Blood Cancer 53:386-391, 2009

80. Meneses $K$, McNees $P$, Azuero A, et al: Development of the Fertility and Cancer Project: An Internet approach to help young cancer survivors. Oncol Nurs Forum 37:191-197, 2010

81. Hess SL, Jóhannsdóttir IM, Hamre H, et al: Adult survivors of childhood malignant lymphoma are not aware of their risk of late effects. Acta Oncol 50:653-659, 2011

82. Goldfarb JM, Fisher NL, Gillespie L, et al: Partnership for Families program: Philanthropy for in vitro fertilization patients. J Reprod Med 54:548552, 2009

83. Shimizu $C$, Bando $H$, Kato $T$, et al: Physicians' knowledge, attitude, and behavior regarding fertility issues for young breast cancer patients: A national survey for breast care specialists. Breast Cancer [epub ahead of print on January 24, 2012]

84. van den Berg $\mathrm{MH}$, Overbeek $\mathrm{A}$, van der Pal $\mathrm{HJ}$, et al: Using web-based and paper-based questionnaires for collecting data on fertility issues among female childhood cancer survivors: Differences in response characteristics. J Med Internet Res 13:e76, 2011

85. Quinn GP, Vadaparampil ST, Malo T, et al: Oncologists' use of patient educational materials about cancer and fertility preservation. Psychooncology [epub ahead of print on July 14, 2011]

86. Quinn GP, Knapp CA, Malo TL, et al: Physicians' undecided attitudes toward posthumous reproduction: Fertility preservation in cancer patients with a poor prognosis. J Support Oncol 10:160-165, 2012

87. Merrick $H$, Wright $E$, Pacey $A A$, et al: Finding out about sperm banking: What information is available on-line for men diagnosed with cancer? Hum Fertil (Camb) 15:121-128, 2012

88. Edge B, Holmes D, Makin G: Sperm banking in adolescent cancer patients. Arch Dis Child 91: 149-152, 2006

89. Chong AL, Gupta A, Punnett A, et al: A cross Canada survey of sperm banking practices in pediatric oncology centers. Pediatr Blood Cancer 55: 1356-1361, 2010

90. Choi BB, Goldstein M, Moomjy $M$, et al: Births using sperm retrieved via immediate microdissection of a solitary testis with cancer. Fertil Steril $84: 1508,2005$ 
91. Reebals JF, Brown R, Buckner EB: Nurse practice issues regarding sperm banking in adolescent male cancer patients. J Pediatr Oncol Nurs 23:182-188, 2006

92. Keros $V$, Hultenby $K$, Borgstr[UML]om B, et al: Methods of cryopreservation of testicular tissue with viable spermatogonia in pre-pubertal boys undergoing gonadotoxic cancer treatment. Hum Reprod 22:1384-1395, 2007

93. Anderson RA, Wallace WH, Baird DT: Ovarian cryopreservation for fertility preservation: Indications and outcomes. Reproduction 136:681-689, 2008

94. Jadoul P, Dolmans MM, Donnez J: Fertility preservation in girls during childhood: Is it feasible, efficient and safe and to whom should it be proposed? Hum Reprod Update 16:617-630, 2010
95. Cvancarova M, Samuelsen SO, Magelssen $\mathrm{H}$, et al: Reproduction rates after cancer treatment: Experience from the Norwegian radium hospital. J Clin Oncol 27:334-343, 2009

96. Nieman $\mathrm{CL}$, Kinahan $\mathrm{KE}$, Yount $\mathrm{SE}$, et al: Fertility preservation and adolescent cancer patients: Lessons from adult survivors of childhood cancer and their parents. Cancer Treat Res 138:201-217, 2007

97. Fallat ME, Hutter J: Preservation of fertility in pediatric and adolescent patients with cancer. Pediatrics 121:e1461-e1469, 2008

98. National Cancer Institute: FDA approval for bevacizumab. http://www.cancer.gov/cancertopics/ druginfo/fda-bevacizumab

99. Apperley $\mathrm{J}$ : CML in pregnancy and childhood. Best Pract Res Clin Haematol 22:455-474, 2009
100. Mead $H$, Cartwright-Smith $L$, Jones $K$, et al: Racial and Ethnic Disparities in U.S. Health Care: A Chartbook. New York, NY, Commonwealth Fund, 2008

101. Howlader $N$, Noone AM, Krapcho $M$, et a (eds): SEER Cancer Statistics Review, 1975-2009 (vintage 2009 populations), National Cancer Institute. http://seer.cancer.gov/csr/1975_2009_pops09/

102. Smedley BD, Stith AY, Neson AR: Confronting Racial and Ethnic Disparities in Health Care. Washington, DC, National Academies Press, 2003

103. American College of Physicians: Racial and Ethnic Disparities in Health Care, Updated 2010. Philadelphia, PA, American College of Physicians, 2010

\section{JOP Online Works for You}

- Select articles are available weeks before they appear in print

- Access to current and archival issues

- ASCO Clinical Guideline Summaries

- Links to citations from 1,758 HighWire-hosted journals

- Customized e-mail alerts and RSS feeds of newly published articles

- Specialized practice resources and tools for enhancing quality of care

- Centralized job search through the online Career Center

Visit jop.ascopubs.org to see what JOP online has to offer. 


\section{Acknowledgment}

The Panel thanks the following for their thoughtful reviews of drafts of the guideline update: Maura Dickler, MD, Scott Gilbert, MD, Andrew Loblaw, MD, and the American Society of Clinical Oncology Clinical Practice Guideline Committee. The Panel also thanks Sarah R. Arvey, $\mathrm{PhD}$, Emily E. Eargle, MSW, Ashley M. Koenings, BS, and Joyce D. Reinecke, JD, of the LIVESTRONG Foundation for their contributions to Data Supplements 5 and 6.

\section{Appendix}

Table A1. Fertility Preservation for Patients With Cancer Guideline Update Panel Members

\begin{tabular}{ll}
\hline \multicolumn{1}{c}{ Member } & \multicolumn{1}{c}{ Affiliation/Institution } \\
\hline Kutluk Oktay, MD, Co-Chair & Innovation Institute for Fertility Preservation, New York Medical College, Rye and New York City, NY \\
Alison W. Loren, MD, Co-Chair & Perelman School of Medicine at the University of Pennsylvania, Philadelphia, PA \\
Lindsay Nohr Beck & LIVESTRONG Foundation's Fertile Hope Program, Austin, TX \\
Lawrence Brennan, MD & Oncology Hematology Care, Crestview Hills, KY \\
Anthony J. Magdalinski, DO & Private practice, Sellersville, PA \\
Ann H. Partridge, MD & Dana-Farber Cancer Institute, Boston, MA \\
Gwendolyn Quinn, PhD & Moffitt Cancer Center, Tampa, FL \\
W. Hamish Wallace, MD & Royal Hospital for Sick Children, Edinburgh, United Kingdom \\
\hline
\end{tabular}

